\title{
Living on the Edge: Re-shaping the Interface of Synthetic Biology and Nanotechnology
}

\author{
Shang-Jung Wu and Ardemis A. Boghossian*
}

\begin{abstract}
A new team of researchers at EPFL is taking an 'anti-disciplinary' approach to creating optical devices. These devices take advantage of the synergy in tuning both nano- and bio-material properties, coupling the advantages of two growing, albeit traditionally distinct, fields. With applications spanning from biosensing and microarray assays to living photovoltaics, the Laboratory of NanoBiotechnology (LNB) is uncovering an unexplored space for the next generation of chemical analytics and light-harvesting technologies.
\end{abstract}

Keywords: Bioengineering · Nanotechnology · Protein engineering · Single-walled carbon nanotube (SWCNT) · Synthetic biology

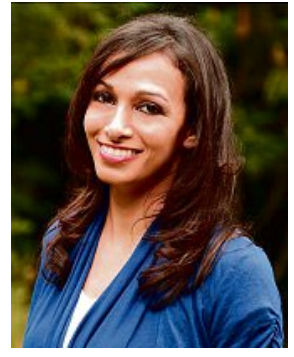

Ardemis Boghossian was born in the United States in Detroit, Michigan. She received her B.Eng. in Chemical Engineering from the University of Michigan in Ann Arbor in 2007. She pursued her graduate studies as a National Defense Science \& Engineering Graduate (NDSEG) fellow at the Massachusetts Institute of Technology (MIT) under the supervision of Michael S. Strano. Her PhD focused on engineering nanoparticles that interface with lightharvesting, biological constructs to enhance solar energy conversion. She also developed algorithms for quantifying stochastic fluctuations in fluorescence from single-molecule, nanotube-based sensors.

${ }^{\star}$ Correspondence: Prof. Dr. A. A. Boghossian Institute of Chemical Sciences and Engineering (ISIC) École polytechnique fédérale de Lausanne (EPFL) $\mathrm{CH}-1015$ Lausanne

E-mail: ardemis.boghossian@epfl.ch
After obtaining her PhD in 2012, she continued her research career in the laboratory of Frances H. Arnold at the California Institute of Technology (Caltech). As a National Institutes of Health (NIH) postdoctoral fellow, she acquired skills in protein engineering that allowed her to biologically engineer cells that can electronically interface with electrodes.

In 2015, Prof. Boghossian became a tenure-track Assistant Professor within the Institute of Chemical Sciences and Engineering (ISIC) at the École polytechnique fédérale de Lausanne (EPFL), where she is currently the Principle Investigator of the Laboratory of NanoBiotechnology (LNB).

Bioengineering is the synthetic biologist's approach to engineering new materials. It has allowed researchers to overcome billions of years of evolution to create biomolecules, such as proteins, that have been re-programmed to interface with synthetic materials or optimized to bind analytes through interactions not found in nature. The ability to modulate protein binding has led to significant advancements in topics ranging from biosensors ${ }^{[1]}$ and pharmaceuticals $^{[2]}$ to remediation ${ }^{[3]}$ and biofuels. ${ }^{[4]}$ Equipped with mechanisms of unparalleled molecular recognition and autonomous fault-tolerance, biological constructs offer cost-effective approaches for manufacturing intricate molecular motifs. However, the commercial development of protein-based constructs for light-harvesting and optical sensing applications has been hindered by protein instabilities due to photodegradation and photobleaching. Many proteinbased optical sensors, for example, rely on fluorophores that photobleach, which limit sensor lifetime. Their fluorescence emissions at visible wavelengths overlap with tissue absorption, further limiting applications for in vivo optical sensing. Similarly, biophotovoltaic devices that rely on lightharvesting capabilities found in nature can only access a fraction of the solar energy spectrum, reducing device efficiencies. ${ }^{[5]}$

Inorganic nanomaterials, on the other hand, demonstrate enhanced photophysical properties that enable optical devices with unprecedented optoelectronic properties. ${ }^{[6]}$ Nanotechnology has realized hot carrier and multiple exciton generating solar cells with theoretical maximum efficiencies of $66 \%{ }^{[7]}$ and $44 \% .{ }^{[8]}$ Nanoscale quantum confinement in particular has enabled tunable, photostable fluorophores that emit light that is distinct from the visible wavelengths typically absorbed by biological tissue. Combined with the ability to detect single molecules, ${ }^{[9]}$ these fluorophores offer a nearly ideal platform for in vivo optical sensing. Unfortunately, these synthetic materials lack the inherent binding specificity and tunability that is needed to develop selective sensors. Furthermore, the cost-effective manufacturing of quantum devices places stringent requirements on minimizing the variability of the critical dimension of the nanostructure.

In a sense, the advantages and disadvantages posed by the fields of synthetic biology and nanotechnology are highly complementary. Solar cells that benefit from robust, biological light-capturing mechanisms suffer from irreversible photo-degradation $^{[10]}$ - a drawback that can be addressed with regenerative, photoprotective nanoparticles. ${ }^{[11]}$ Similarly, fluorescence-based nano-sensors that have indefinite photostabilities ${ }^{[12]}$ can benefit from the selectivity imparted to them by 
enzymes. Engineered proteins have been interfaced with nanomaterials before, where properties such as increased surface area to volume ratios and enhanced permeabilities have been used to develop targeted drug delivery mechanisms.[13] However, biologically engineered platforms that capitalize on nanoscale properties such as tunable light-absorption and photostable luminescent emissions, which are particularly beneficial to the energy and sensing fields, remain under-explored.

To exploit this untapped synergy, Prof. Ardemis Boghossian has assembled a highly distinguished team of interdisciplinary researchers seeking to overcome 'anti-disciplinary' challenges. With backgrounds spanning material science, physics, molecular biology, biotechnology and bioengineering, chemistry, and chemical engineering, this interdisciplinary ensemble of experiences form the core expertise of the LNB. Through its focal points in nano-optoelectronics and bioengineering, the LNB is forging new biophysical methods to not only create hybrid materials, but also to establish unorthodox bioengineering platforms that can be used to overcome otherwise intractable impediments in the field. In particular, the laboratory specializes in platforms that benefit from singlewalled carbon nanotube (SWCNT) optoelectronic properties for both biosensing and light-harvesting applications.

\section{Why Single-walled Carbon Nanotubes?}

SWCNTs offer a collectively unique combination of advantageous optical and chemical properties. ${ }^{[12]}$ SWCNTs structurally resemble rolled up sheets of graphene that form cylindrical tubes of carbon at- oms. They are approximately $1 \mathrm{~nm}$ in diameter and usually a few hundred nanometers in length, though their lengths may vary from $100 \mathrm{~nm}$ up to several centimeters. The different directions in which the graphene sheet is rolled results in different SWCNT chiralities that vary in diameter and bandgap. Each chirality is assigned a pair of $(n, m)$ indices that is indicative of the direction in which the graphene sheet is rolled to form that chirality. SWCNTs may either be metallic or semiconducting, depending on the specific chirality's bandgap. SWCNTs demonstrate broad absorption ranging from UV to visible wavelengths (Fig. 1a). The broad absorption of semiconducting chiralities throughout the visible region has been particularly beneficial in engineering tandem solar cells. ${ }^{[14]}$ The semiconducting SWCNTs also benefit from autofluorescence, as they are able to absorb visible light upon $\mathrm{E}_{22}$ excitation, and the resulting $\mathrm{E}_{11}$ fluorescence emissions occur in the near-infrared (Fig. 1b).

The near-infrared fluorescence emissions are especially useful for in vivo imaging and sensing applications. Blood and tissue absorb light at visible wavelengths below $700 \mathrm{~nm}$, and water absorption occurs at infrared wavelengths above 1300 $\mathrm{nm}$. Biological tissue is thus optically transparent to SWCNT fluorescence at wavelengths between 700 and $1300 \mathrm{~nm}$, enabling optical in vivo applications that are otherwise not possible using conventional visible fluorophores. Furthermore, these fluorescence emissions are indefinitely photostable; they do not photobleach. This photostability allows for continuous, quantitative imaging and sensing applications.

In addition to tissue transparency and photostability, SWCNT fluorescence is sensitive to the local environment. As shown in Fig. 1c, a suspended mixture of SWCNT chiralities contains several fluorescence peak maxima at specific excitation and emission wavelengths that correspond to individual chiralities. The intensity and location of these peaks vary with factors such as $\mathrm{pH},{ }^{[15]}$ dielectric constant, ${ }^{[16]}$ and charge transfer to and from the SWCNT, ${ }^{[6]}$ which may, in turn, result in an increase or decrease in fluorescence intensity or a red- or blue-shifting of the fluorescence emissions. In fact, the fluorescence is so sensitive to the SWCNT environment that it is perturbed by the adsorption and desorption of individual molecules, enabling sensors with single-molecule sensitivities. ${ }^{[9 a, b, d]}$ The fluorescence response is chirality-specific, and each of the fluorescence peaks may undergo a distinct response to perturbations in the surrounding environment. This decoupled behavior can be used for multimodal sensing applications, where different chiralities are sensitive to the presence of different analytes, and a mixture of analytes can be analyzed by simultaneously monitoring the response of individual peaks. [9c]

Though SWCNT fluorescence is sensitive to the nanotube environment, it is not inherently selective. SWCNT surfaces must be specifically engineered for selectivity towards specific analytes when devloping optical sensors. The hydrophobic, electron-rich surfaces are amenable to a variety of conjugation schemes, resulting in tunable surface chemistries. Since covalent functionalization disrupts their electronic properties, SWCNTs must be non-covalently conjugated to biological or chemical moieties to preserve their optical characteristics. They have been conjugated to a variety of wrappings, such as synthetic polymers, as well as biomolecules such as deoxyribonucleic acid (DNA), lipids, and
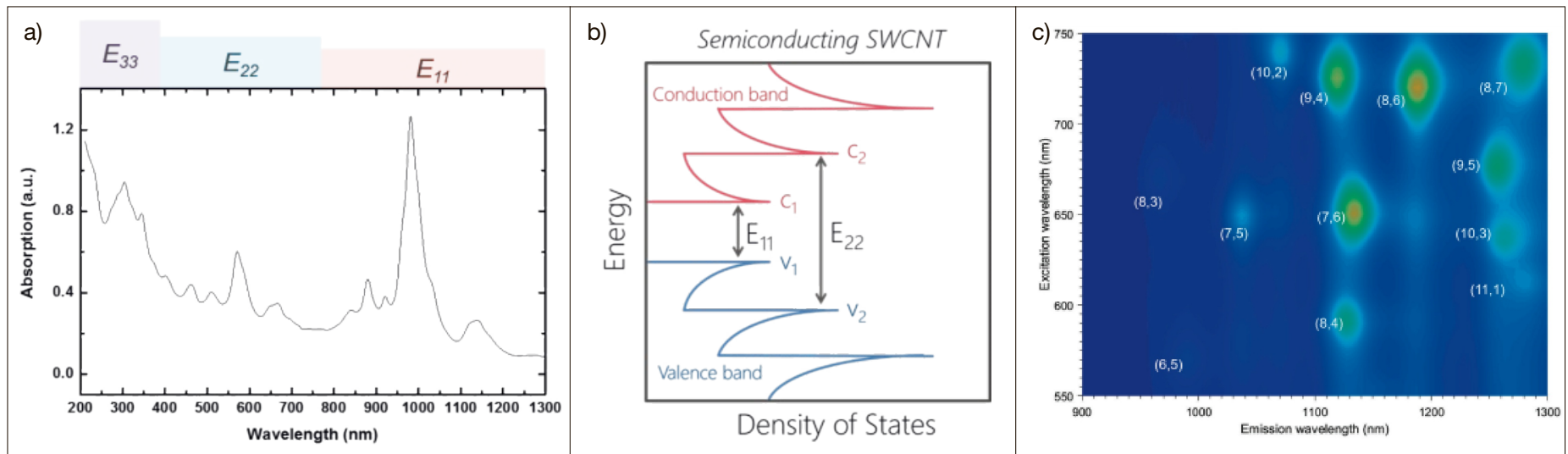

Fig. 1. Fluorescence from semiconducting SWCNTs. (a) An absorption spectrum of a sodium cholate (SC)-suspended aqueous mixture of SWCNT chiralities shows absorption from the UV to near-infrared range. Three regions in the absorption spectrum that correspond to three different types of electronic transitions $\left(\mathrm{E}_{11}, \mathrm{E}_{22}\right.$, and $\mathrm{E}_{33}$ transitions) are shown. (b) The SWCNT band structure consists of sharp van Hove singularities that are characteristic of one-dimensional exciton confinement. Visible light $(500-900 \mathrm{~nm})$ is absorbed upon $\mathrm{E}_{22}$ excitation, and near-infrared light is emitted during $\mathrm{E}_{11}$ relaxation. (c) An excitation-emission plot of a SC-suspended aqueous mixture of SWCNT chiralities produced using high-pressure carbon monoxide (HiPco) reveals several peaks corresponding to different chiralities. The specific chiralities are labeled according to their $(n, m)$ indices. 
proteins, that can impart the SWCNT with selectivity. ${ }^{[9 a, b, d, 17]}$ The specific mechanism that provides these wrappings with selectivity depends on the wrapping, and in most cases, remains unclear. For example, specific DNA wrappings have been hypothesized to behave as molecular sieves, allowing only molecules below a certain size threshold to penetrate the wrapping and interact with the nanotube. ${ }^{[0 b]}$ Protein-based wrappings can trigger an optical SWCNT response based on protein activity, where a protein conformational change or reactivity alters the SWCNT environment in a manner that affects SWCNT fluorescence.

Biomolecules such as DNA and proteins offer distinct advantages in engineering selectivity since they benefit from the engineerability and tunability in specificity that have been utilized by bioengineers for decades. In engineering polymer-wrapped SWCNTs, SWCNTs are noncovalently conjugated to a variety of synthetic polymers that are then screened against a collection of analytes. ${ }^{[17 b]}$ This empirical approach to engineering wrappings is circumvented with biomolecules, which have a pre-determined, natural specificity to analytes that can be tuned through bioengineering. However, despite this versatility, SWCNT conjugation has largely been confined to wildtype proteins and oligonucleotides that have not benefited from the optimization and tunability offered through bioengineering. Uniquely equipped with both synthetic biology and SWCNT optoelectronic tools, the LNB focuses on developing this untapped potential for the range of applications summarized in Fig. 2. Given the aforementioned synergy in opti-

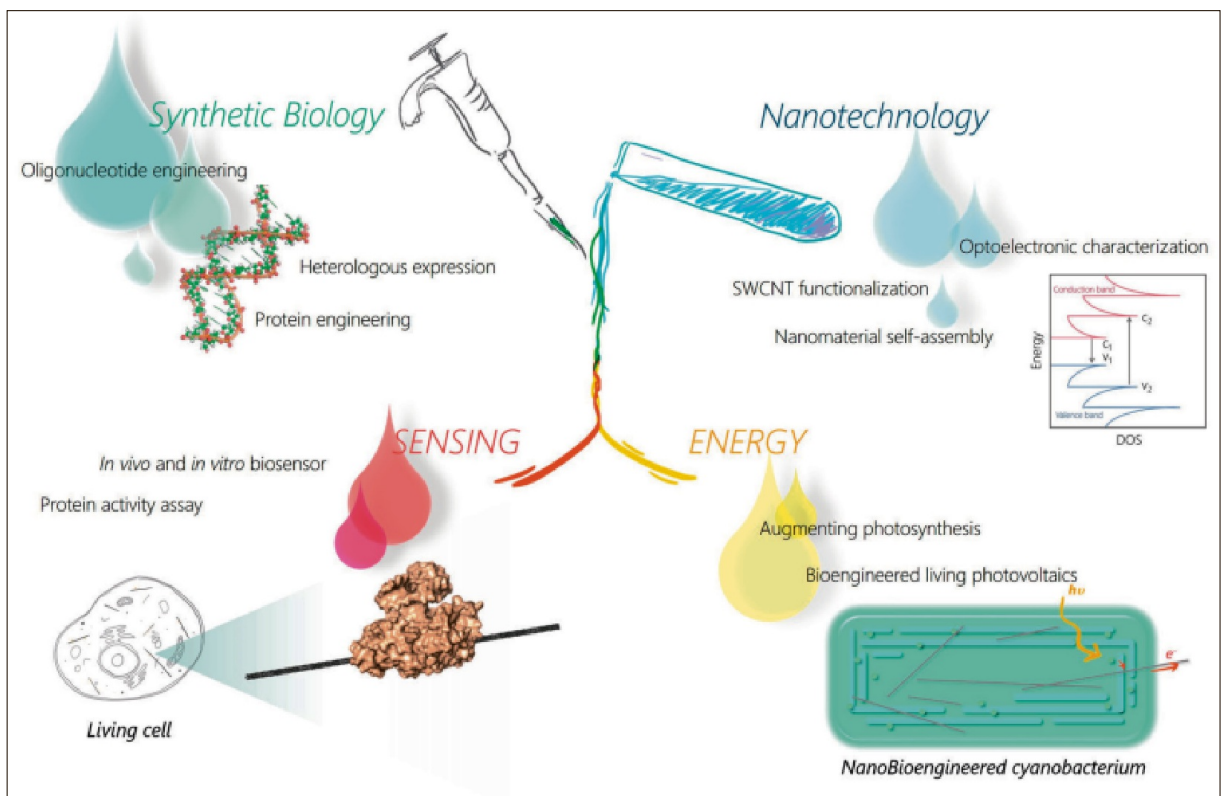

Fig. 2. Overview of LNB research. Synthetic biology techniques (upper left) and nano-optoelectronic tools (upper right) form the crux of the LNB toolkit. The LNB uses these tools to engineer optical sensing materials (lower left) based on SWCNT fluorescence and solar energy materials (lower right) that benefit from photosynthesis.

cal applications, the lab currently focuses on two areas of research: optical sensing and energy materials.

\section{SWCNT-based Optical Sensing}

The LNB sensors largely focus on coupling SWCNT fluorescence with biomolecules such as oligonucleotides and proteins (Fig. 3). In contrast to conventional synthetic polymers, where different poly- mers each require a unique synthesis procedure, bioengineering techniques such as protein mutagenesis and polymerase chain reaction (PCR) are capable of producing thousands of biomolecular variants in a parallel, scalable manner. Combined with a relatively high-throughput approach to measuring SWCNT fluorescence 96-well plates, this platform provides an effective way to screen a large number of variants over a short period of time. A two-fold perspective on engineering applications exists:

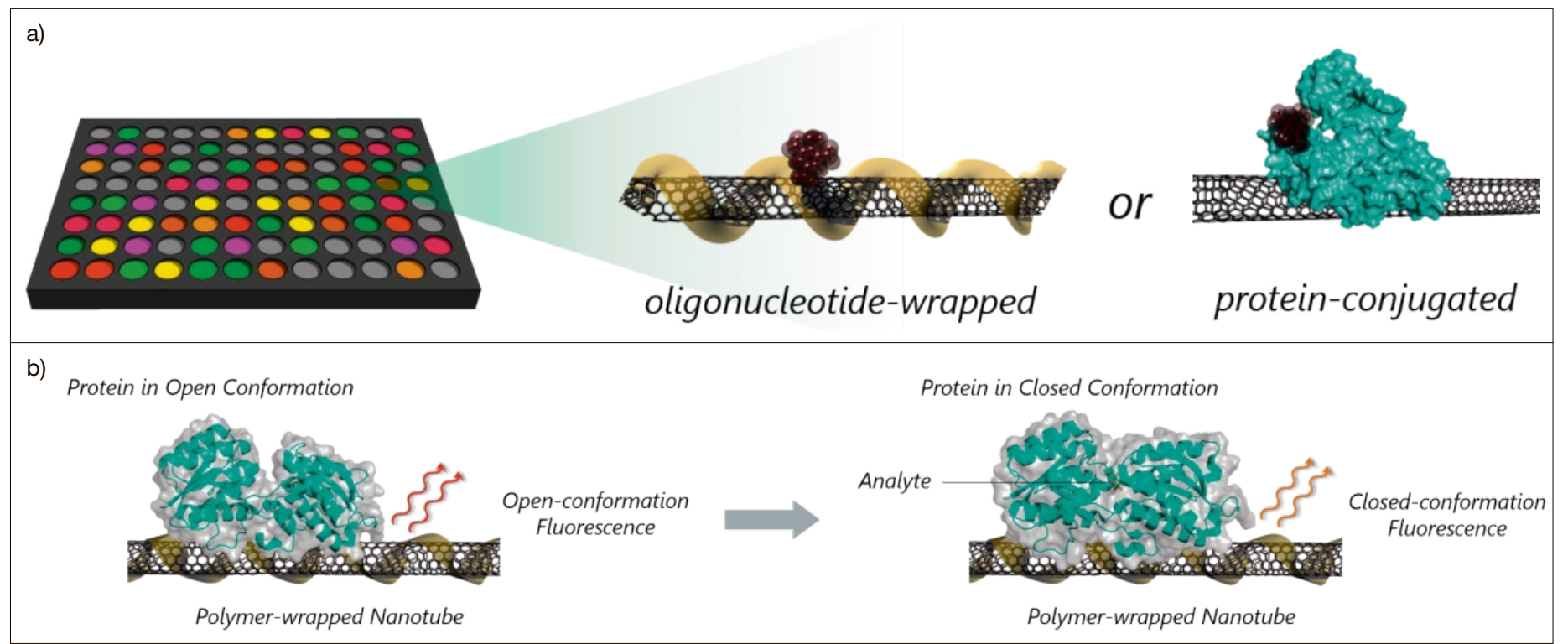

Fig. 3. High-throughput biosensing platform. (a) A custom-built setup allows SWCNT fluorescence to be assayed in 96-well plates. The 96-well plates can be used to assay either oligonucleotide-wrapped (middle) or protein-conjugated (right) SWCNTs. The oligonucleotide (yellow) and protein (cyan) are shown binding their target analyte (dark purple). (b) One possible sensing mechanism based on a protein conformation change is shown. A binding protein (blue) is conjugated to a polymer-wrapped nanotube (left). Binding of the analyte to the protein (right) results in a protein conformational change that affects nanotube fluorescence. 
from a materials science perspective, this technique can be used to develop optical sensors with tunable sensitivities towards specific analytes; from a bioengineering perspective, this technique provides a relatively high-throughput approach to assaying protein activity. Both perspectives and the applications they enable are discussed below.

\section{Bioengineered Optical Sensors}

Optical SWCNT-based sensors developed by the LNB use proteins with known specificities conjugated to SWCNTs. SWCNT fluorescence is then used as an optical means of detecting analytes that interact with the immobilized proteins. The protein interacts with the biomolecule of interest, and a change in protein conformation or activity changes the SWCNT environment in a manner that perturbs fluorescence. Such a platform not only benefits from SWCNT optical properties, such as photostability, in vivo optical transparency, and single-molecule detection limits, but also from the biomolecular specificity of proteins, enabling SWCNTs sensors with unprecedented 'smart' wrappings that are pre-configured to recognize identified analytes of interest.

The strict use of wildtype proteins in engineering these SWCNT-based optical sensors, however, severely restricts the functionality of these sensors. Proteins have undergone billions of years of evolution to yield highly specialized molecular machinery that has been optimized for a specific function inside or on the surface of a cell. When wildtype proteins are placed in a non-natural physiochemical environment interfacing dielectric, semiconducting, or metallic surfaces, they may need to be re-engineered to perform optimally in their new environment. Protein engineering allows researchers to tune or even altogether change protein behavior by modifying the amino acid sequence of a protein. It has been largely used to engineer enzymes that catalyze non-natural chemistries by altering enzyme specificity, changing the product distribution, or creating a new metabolite. Protein engineering has also been used to tune protein behavior, allowing proteins to more effectively interact with and function in the presence of nanomaterials. ${ }^{[18]}$

With protein engineering techniques at hand, the LNB is developing a new generation of NanoBiOptic devices - devices that benefit from the synergistic combination of nanomaterial-biological hybrids - for sensing applications using bioengineered proteins. Engineering the proteins to behave optimally in the presence of SWCNTs may lead to not only improved sensor sensitivities and response times, but also enhanced protein stabilities. The means by which bioengineering is used to improve sensors depends on the protein and the sensing mechanism. For example, a SWCNT-based sensor whose optical response is triggered by a protein conformation change would benefit from endeavors that focus on increasing the conformation change to enhance the fluorescence response. Such an approach can be used to engineer current protein-based, SWCNT glucose sensors that suffer from sensitivity limits above those needed for the physiological detection of blood sugar.[17a] The creation of an implantable, in vivo optical sensor capable of continuously monitoring blood sugar exemplifies just one of many point-of-care diagnostic tools enabled by coupling SWCNT fluorescence with engineered proteins.

In addition to proteins, oligonucleotides also benefit from tunability enabled through bioengineering. DNA-based sensors have been used to detect a variety of analytes, including nitric oxide (NO), ${ }^{[9 b]}$ hydrogen peroxide $\left(\mathrm{H}_{2} \mathrm{O}_{2}\right),{ }^{, 9 c]}$ and dopamine.[9a] Unlike proteins, where analyte specificity is identified a priori, an empirical approach is used to engineer these DNA-wrapped sensors; a variety of DNA sequences are wrapped around the SWCNTs and screened against a collection of analytes of interest. As with synthetic polymers, the underlying mechanism behind the DNA-based selectivity for these sensors remains elusive. However, in contrast to most polymer synthesis procedures, a large number of DNA variants can be produced in a single PCR vial. For example, a single PCR reaction can be used to create a collection of DNA variants with random mutations throughout the template strand. Since sensor selectivity is determined by the DNA sequence, this technique provides a large library of SWCNT wrappings that can be screened. In this platform, the SWCNT conjugation and fluorescence screening, rather than DNA synthesis, would limit throughput.

A more rational approach can be taken to engineer SWCNT-based sensors that are selective for specific DNA sequences. In such cases, SWCNTs are wrapped with complementary oligonucleotide sequences that can bind target DNA. Previous studies have shown selective binding affinities that are sensitive enough to discern single nucleotide polymorphisms (SNPs). ${ }^{[17 c]}$ To date, this technology has been largely used to demonstrate proof-of-concept principles for identifying SNPs in the genome that may be associated with gene malfunction and several complex diseases. An application that has remained largely unexplored by this technology is the detection of microRNAs which constitute a class of short, non-coding RNAs that play a key role in regulating gene expression. MicroRNAs have recently been implicated as biomarkers and potential therapeutic targets for a variety of diseases, including heart disease, liver disease, and different kinds of cancer. ${ }^{[19]}$ Conjugating SWCNT with oligonucleotides with engineered specificities towards microRNA biomarkers offers one promising prospect for diagnosing and monitoring disease progression in vivo.

The nearly limitless possibilities enabled by manipulating DNA sequences can be further expanded with artificial oligonucleotides. Recent years have seen a boost in the development of synthetic oligonucleotides that are capable of binding complementary DNA and/or RNA strands.[20] Synthetic oligonucleotides may benefit from thermostability and in some cases, improved binding affinities towards their complementary strands. This disparity in binding chemistry compared to conventional oligonucleotides suggests that synthetic nucleotides may contribute to a distinct sensing mechanism on the SWCNT surface. This possibility unlocks an unexplored space for engineering SWCNT wrappings.

\section{Optical Bioassays}

A complementary outlook on the aforementioned sensing technologies suggests that SWCNTs can be used to assay for protein and bio-activity. For example, a perturbation in SWCNT fluorescence can be used to verify that a particular protein is functional or that a particular nucleotide sequence is capable of either identifying a specific sequence in the genome or, in the case of aptamer-protein interactions, binding a specific protein. Coupled to a relatively high-throughput approach for conjugating and measuring SWCNT fluorescence, this platform can be used as a powerful technique for bioengineering applications.

Bioengineering itself has become indispensable in shaping an array of fields spanning biosensing, ${ }^{[1]}$ genome editing, ${ }^{[21]}$ drug discovery, ${ }^{[22]}$ and biocatalysis. ${ }^{[23]}$ Proteins, for example, consist of a sequence of amino acids whose activities can be tuned or even altogether altered simply by mutating an individual amino acid. The ability to tune protein specificity, reactivity, and stability is largely a consequence of keen design strategies that circumvent limited protein knowledge. Unlike materials engineering, where a foundational understanding of the structure-function relation is necessary for altering material properties in a rational manner, bioengineering must address additional challenges since it often requires engineering a material with a structure-function relation that is not comprehensively defined.

One approach to engineering proteins when little to no knowledge on protein 
structure exists is through directed evolution. Directed evolution circumvents the challenges of having limited information on the structure-function relation of a protein by applying a large-scale, trial-and-error approach to identify modifications, or mutations, that are beneficial to the desired protein function. ${ }^{[24]}$ A generalized schematic of this technique is shown in Fig. 4. Mutations are introduced in the original, or wild-type, protein by altering the nucleotide sequence of the DNA to create a collection of proteins with different amino acid sequences known as the protein library. Using a medium- to high-throughput assay, the proteins are tested and proteins that demonstrate the desired properties enter a second round of evolution, where they are once more mutated to create libraries, and new mutants with improved activities are isolated. This process is repeated, iteratively using the best mutant(s) from the previous round as a template for the next round.

The assay used to assess protein activity is key to designing a directed evolution platform. When screening is used to identify desired mutants, the library of mutants is assayed to measure protein activity, and candidates with the desired mutations are then selected. For example, if a protein is being engineered for improved catalytic activity at a nanoparticle interface, one approach would be to screen for protein activity in the presence of the nanoparticle and select the protein mutants that demonstrate improved activity compared to that of the wild-type protein in the presence of the nanoparticle.

The primary advantage of using directed evolution is that one can engineer a protein without any information on protein structure. By introducing mutations at random and iteratively picking only the proteins with beneficial mutations, this technique overcomes the need to have underlying knowledge of the structure-function relation of a given protein. One key challenge in applying directed evolution to a protein is designing a screening method that can assay for the desired property in a scalable, high-throughput manner. For a protein containing 100 amino acids, there are a total of $20^{100}$ possible combinations of amino acid sequences - a number that far exceeds the number of proteins that can be screened in even the highest throughput applications. In some cases, screening several thousand variants is sufficient for identifying beneficial mutations. In practice, traditional characterization tools, such as mass spectroscopy (MS), nuclear magnetic resonance (NMR) spectroscopy, and high pressure liquid chromatography (HPLC) have limited throughput. Colorimetric assays that rely on absorption or fluorescence, on the other hand,

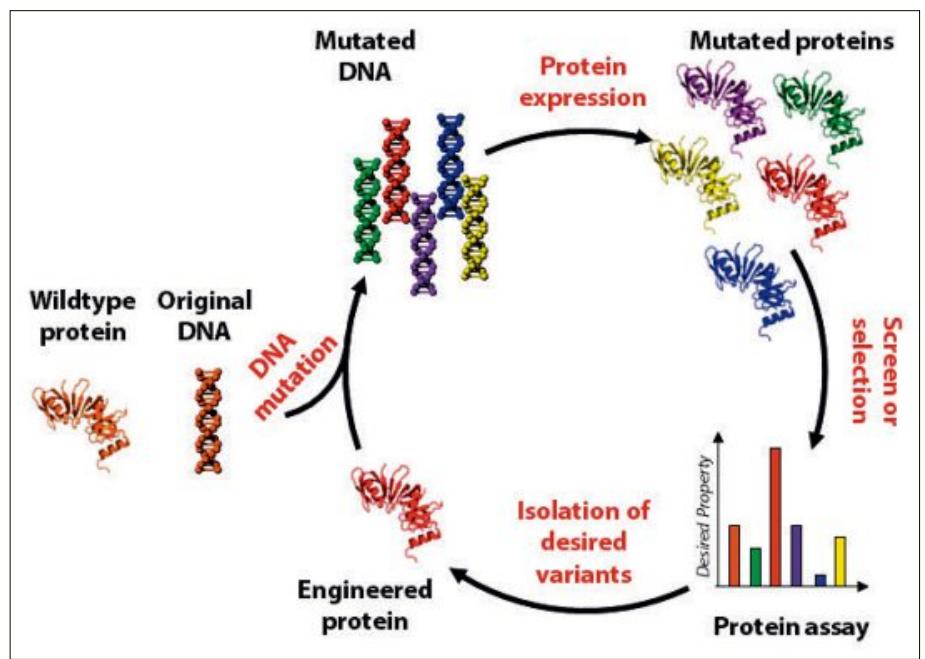

Fig. 4. Schematic outline of a representative directed evolution approach. DNA encoding wild-type protein is mutated, and mutated proteins are expressed. The mutants may undergo a screening process, where cells or proteins with desirable properties survive or remain, or a screening process, where each protein is assayed for the desired property. The protein(s) that demonstrate the desired behavior is selected and used as a template for the next round of mutation. The process is continued until protein activity is optimized. In this schematic, different colors represent different mutants.

offer a scalable screening approach that can be applied to either 96-well plates or fluorescence-activated cell sorting (FACS) platforms that are amenable to medium to high throughput screening.

The LNB is developing SWCNT-based platforms for assaying proteins that are otherwise not amenable to standard colorimetric assays. Two particular advantages for using SWCNT fluorescence for screening are the fluorescence sensitivity and near-infrared emissions. As discussed above, SWCNT fluorescence is very sensitive to the SWCNT environment and capable of responding to single-molecule binding events. This extreme sensitivity allows the SWCNT to respond to slight changes in its immediate environment, including conformational changes that can be used to screen for protein activity or protein binding. The near-infrared emissions have the added benefit of enabling a sensing mechanism that does not interfere with protein activity. Many proteins fluoresce or absorb light in the visible spectra. Some proteins, such as opsins, even rely on visible light to trigger activity. Traditional fluorophores and dyes that are used in colorimetric protein assays would have their optical signal compromised by protein interference or, in cases where the protein relies on visible light for activation, would interfere with the protein activity that is being measured. Because the near-infrared fluorescence emissions are distinct from visible wavelengths absorbed and emitted by biological fluorophores, this proposed platform offers a promising approach to engineering light-sensitive proteins.
Beyond directed evolution, this platform may be applied to screen binding interactions between various biomolecules. For example, enzyme-linked immunosorbent assay (ELISA) is a multi-step colorimetric screen for quantifying antibody-antigen interactions. In an ELISA assay, antigens are immobilized on a solid surface and exposed to antibodies that are linked to an enzyme. After incubation, the surface is washed, leaving the enzyme-conjugated antibodies bound to the immobilized antigen. An enzymatic substrate is added to produce an optical signal that can be used to quantify the antigen concentrations. In this example, SWCNTs would offer a promising alternative to quantifying the antibody concentrations, since fluorescence emissions from antigen-decorated, immobilized SWCNTs can be directly used to monitor binding in the absence of an added enzymatic substrate. This platform would also obviate the need to conjugate the antibodies to enzymes. In addition to antibody-antigen interactions, oligonucleotide hybridization and protein-aptamer interactions may also be monitored using this approach.

\section{Nanobiohybrids for Light- harvesting Applications}

Natural light-harvesting mechanisms such as photosynthesis offer a cost-effective, scalable alternative to harnessing solar energy. The autonomous self-replication and self-repair mechanisms enable fault-tolerant designs that facilitate manu- 
facturing and maintenance. Furthermore, photosynthetic organisms sequester carbon from the atmosphere, and the negative carbon footprint helps partially offset the carbon surplus responsible for greenhouse gas effects. A common misconception of photosynthesis is that it is inherently inefficient. However, the Shockley-Queisser efficiency limit of photosystem II (PSII), the light-sensitive protein complex involved in water splitting, is about $24 \%$, comparable to the maximum efficiencies achieved with silicon-based devices.[25] Unfortunately, when actually incorporated into a biophotovoltaic, wildtype photosynthetic organisms have device efficiencies that are less than $1 \% ;[26]$ subsequent metabolic losses and energy dissipation mechanisms diminish overall cell efficiencies.

The seemingly poor design nature has given us for creating photovoltaics is a consequence of the very process responsible for engineering optimized proteins: evolution. Photosynthetic organisms have evolved under the pressure of survival. This means that though their metabolic processes have been engineered to survive competitive environments with limited resources, they have not been optimized to behave as photovoltaics. In fact, photosynthetic organisms that naturally demonstrate photovoltaic behavior would be disadvantaged by exporting charge that they can otherwise use for energy storage. Evolution has even favored species with limited absorption to protect proteins from photodamage. Because of this, despite the $100 \%$ internal quantum efficiency, photosynthetic organisms can only access approximately $45 \%$ of the solar energy spectrum (Fig. 5a). Furthermore, up to $80 \%$ of the energy that is captured by the cell is dissipated as heat or fluorescence.

The LNB uses two complementary approaches to engineering biophotovoltaics: a nano-materials engineering and a bioengineering approach. In the former, photosynthetic organisms are coupled to SWCNTs to create nanobionic organisms with enhanced photosynthetic efficiencies. ${ }^{[27]}$ The latter approach is used to engineer living organisms capable of harnessing solar energy and exporting current. These approaches are discussed in greater detail in the following sections.

\section{Nanobionics for Augmenting Photosynthesis}

The tunable bandgaps possessed by SWCNTs have strong implications for engineering light-harvesting materials. They absorb light over a range of wavelengths throughout the visible region, and the charge separation in semiconducting SWCNTs has been used to create carbonbased photovoltaics. ${ }^{[14]}$ Because each chirality possesses a distinct bandgap and absorption peak, separated chiralities can be assorted in a bandgap-dependent, tandem configuration that improves absorption and conversion efficiencies. ${ }^{[28]}$ The recent discovery of SWCNT up-conversion substantiates the use of SWCNTs for harnessing near-infrared light, a region of the solar spectrum that is largely inaccessible to existing photovoltaics. Despite these advantages, photovoltaics that rely purely on SWCNT photoabsorption and charge separation suffer from the limitations in manufacturing scalability and high costs factors that serve as advantages in biological light-harvesting devices.
Coupling the light-harvesting capabilities of SWCNTs with the fault-tolerance and energy storage machinery of photosynthetic systems establishes a nanobionic platform with a synergistic approach for solar energy conversion. Photosynthetic organisms, such as cyanobacteria, have been integrated into biophotovoltaic devices before, where they are placed on an anode and produce a current when illuminated, consuming water and carbon dioxide in the process. When augmented with nanomaterials that can broaden the absorption spectra, such as SWCNTs (Fig. 5a), these devices may undergo enhanced photoconversion efficiencies through energy transfer. Nanoparticle-based platforms have been used in previous studies to boost photosynthetic yields, though the underlying mechanism for these improvements has yet to be understood. ${ }^{[11,27]}$

The nanobionic platforms developed thus far have largely focused on using chloroplasts, which are photosynthetic organelles, as opposed to isolated protein and thylakoid membranes with poor photostabilities.[29] Although previous endeavors have focused on developing biomimetic self-repair cycles that can regenerate photo-damaged proteins in light-harvesting devices, ${ }^{[29 b]}$ these platforms fail to capitalize on the autonomous regeneration and replication systems that are inherent to whole-cell, photosynthetic organisms such as cyanobacteria. Through nanomaterials engineering, the LNB is developing the next generation of nanobionic lightharvesting platforms, interfacing living organisms with enhanced light-harvesting capabilities enabled through nanotechnology.

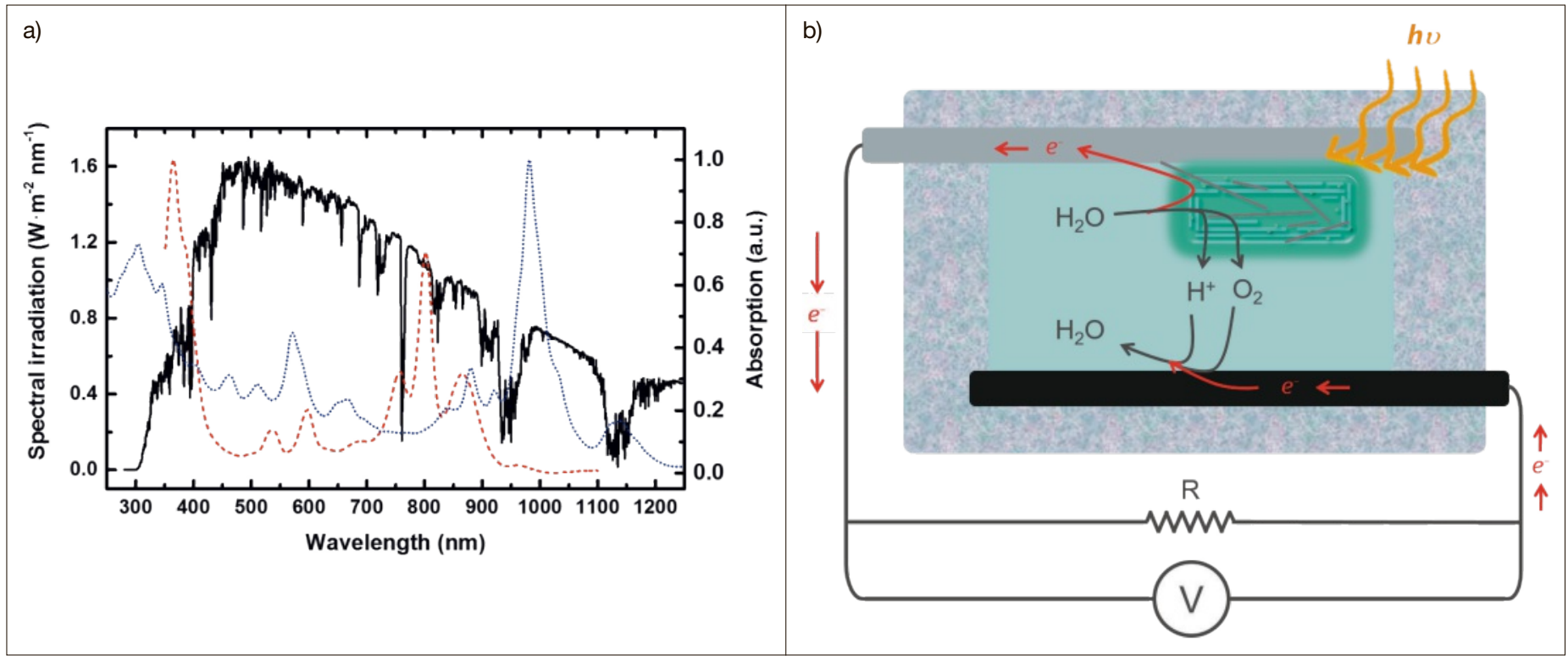

Fig. 5. Engineering improvements in nanobionic energy devices. (a) The solar energy spectrum (black) is compared to the absorption spectra of photosystem II (red) and a mixture surfactant-suspended SWCNTs (blue). Scales for both spectral irradiation (left) and absorption (right) are shown. (b) In cyanobacteria-based photovoltaics, light is absorbed to split water. The extracted electron is shuttled through a series of redox reactions and ultimately used for chemical energy storage. The electron extraction step is limiting and may be improved through bioengineering. 


\section{Bioengineering Living Photovoltaics}

With a strong foothold in nanomaterials engineering, the LNB also leverages its synthetic biology background to bioengineer living organisms, such as cyanobacteria, re-programming them to behave as living photovoltaics (Fig. 5b). Current cyanobacteria-based photovoltaics suffer from inefficient charge extraction. The insulating outer membrane of these cells have been specifically engineered by nature to retain charge. In the absence of conductive pili or extracellular redox proteins, artificial mediators are typically required to efficiently extract charge. The addition of exogenous mediators compromise the scalability and stability of such devices for commercial application. Even in the presence of exogenous mediators, the maximum power output of these devices falls far below those obtained from synthetic photovoltaics.

A promising approach to overcoming the limitations of using natural light-harvesting systems is to genetically modify cyanobacteria to export electrons, allowing them to become living solar cells. The photovoltaic behavior will become innate to the cell, integrating electron-exporting capabilities into the cell's DNA and obviating the need to enhance performance with added mediators.

\section{Summary and Outlook}

The fields of synthetic biology and nanotechnology have each fueled some of the most creative technologies in recent decades. Whether it be manipulating individual atoms to build new materials or constructing a living, microbial factory to synthesize new chemicals, the scientific community has access to an unprecedented number of tools for realizing technologies that were once only imaginable. Biology occurs at the nanoscale, and the ability to simultaneously engineer nanomaterials and biological constructs would further expand the realm of possibilities discussed in Richard Feynman's seminal lecture, 'There's Plenty of Room at the Bottom'. The LNB seeks to couple the abyss of possibilities enabled by nanotechnology with the nearly infinite protein and DNA sequence space.

The LNB specifically focuses on optical platforms that capitalize on the distinct advantages offered by the biological and synthetic worlds. SWCNTs, which demonstrate beneficial optoelectronic properties, are conjugated to biomaterials, such as proteins and DNA, with exquisite molecular recognition to create optical sen- sors and platforms for studying proteinprotein, oligonucleotide-oligonucleotide, and oligonucleotide-protein interactions. SWCNTs also form the basis of nanoparticle-enhanced photosynthesis, which can be used to develop improved biophotovoltaics. By genetically re-purposing living, photosynthetic organisms for photovoltaic applications, the LNB leverages its foundations in bioengineering to further improve biophotovoltaics.

The LNB is uniquely equipped with both SWCNT optoelectronic and bioengineering capabilities. It houses Switzerland's only custom-built optical setup for near-infrared SWCNT fluorescence spectroscopy alongside molecular biology equipment. With these tools at hand, it explores anti-disciplinary approaches to engineering new technologies, striving to further expand the already plentiful space at the bottom.

\section{Received: October 5, 2016}

[1] J. Zhang, R. E. Campbell, A. Y. Ting, R. Y. Tsien, Nat. Rev. Mol. Cell Biol. 2002, 3, 906

[2] D.-K. Ro, E. M. Paradise, M. Ouellet, K. J. Fisher, K. L. Newman, J. M. Ndungu, K. A. Ho, R. A. Eachus, T. S. Ham, J. Kirby, M. C. Y. Chang, S. T. Withers, Y. Shiba, R. Sarpong, J. D. Keasling, Nature 2006, 440, 940.

[3] M. Izallalen, R. Mahadevan, A. Burgard, B. Postier, R. Didonato Jr, J. Sun, C. H. Schilling, D. R. Lovley, Metabolic Engin. 2008, 10, 267.

[4] P. P. Peralta-Yahya, F. Zhang, S. B. del Cardayre, J. D. Keasling, Nature 2012, 488, 320

[5] R. E. Blankenship, D. M. Tiede, J. Barber, G. W. Brudvig, G. Fleming, M. Ghirardi, M. R. Gunner, W. Junge, D. M. Kramer, A. Melis, T. A. Moore, C. C. Moser, D. G. Nocera, A. J. Nozik, D. R. Ort, W. W. Parson, R. C. Prince, R. T. Sayre, Science 2011, 332, 805.

[6] a) P. W. Barone, S. Baik, D. A. Heller, M. S. Strano, Nat. Mater. 2005, 4, 86; b) S. D. Tilley, M. Cornuz, K. Sivula, M. Grätzel, Angew. Chem. Int. Ed. 2010, 49, 6405; c) O. Limaj, D. Etezadi, N. J. Wittenberg, D. Rodrigo, D. Yoo, S.-H. Oh, H. Altug, Nano Lett. 2016, 16, 1502; d) K. Aitola, K. Sveinbjornsson, J.-P. Correa-Baena, A. Kaskela, A. Abate, Y. Tian, E. M. J. Johansson, M. Gratzel, E. I. Kauppinen, A. Hagfeldt, G. Boschloo, Ener. Environ. Sci. 2016, 9,461 .

[7] A. J. Nozik, Physica E: Low-dimensional Systems and Nanostructures 2002, 14, 115.

[8] M. C. Hanna, A. J. Nozik, J. Appl. Phys. 2006, $100,074510$.

[9] a) S. Kruss, M. P. Landry, E. Vander Ende, B. M. A. Lima, N. F. Reuel, J. Zhang, J. Nelson, B. Mu, A. Hilmer, M. Strano, J. Am. Chem. Soc. 2014, 136, 713; b) J. Zhang, A. A. Boghossian, P. W. Barone, A. Rwei, J.-H. Kim, D. Lin, D. A. Heller, A. J. Hilmer, N. Nair, N. F. Reuel, M. S. Strano, J. Am. Chem. Soc. 2011, 133, 567; c) D. A. Heller, H. Jin, B. M. Martinez, D. Patel, B. M. Miller, T.-K. Yeung, P. V. Jena, C. Hobartner, T. Ha, S. K. Silverman, M. S. Strano, Nat. Nano 2009, 4, 114; d) H. Jin, D. A. Heller, M. Kalbacova, J.-H. Kim, J. Zhang, A. A. Boghossian, N. Maheshri, M. S. Strano, Nat. Nano 2010, 5, 302.

[10] A. A. Boghossian, M.-H. Ham, J. H. Choi, M. S. Strano, Ener. Environ. Sci. 2011, 4, 3834.
[11] A. A. Boghossian, F. Sen, B. M. Gibbons, S. Sen, S. M. Faltermeier, J. P. Giraldo, C. T. Zhang, J. Zhang, D. A. Heller, M. S. Strano, Adv. Ener. Mater. 2013, 3, 881.

[12] A. A. Boghossian, J. Zhang, P. W. Barone, N. F. Reuel, J.-H. Kim, D. A. Heller, J.-H. Ahn, A. J. Hilmer, A. Rwei, J. R. Arkalgud, C. T. Zhang, M. S. Strano, ChemSusChem 2011, 4, 848.

[13] A. Arís, A. Villaverde, Trends Biotechnol. 2004 $22,371$.

[14] R. M. Jain, R. Howden, K. Tvrdy, S. Shimizu, A. J. Hilmer, T. P. McNicholas, K. K. Gleason, M. S. Strano, Adv. Mater. 2012, 24, 4436.

[15] M. S. Strano, C. B. Huffman, V. C. Moore, M. J. O'Connell, E. H. Haroz, J. Hubbard, M. Miller, K. Rialon, C. Kittrell, S. Ramesh, R. H. Hauge, R. E. Smalley, J. Phys. Chem. B 2003, 107, 6979.

[16] J. H. Choi, M. S. Strano, Appl. Phys. Lett. 2007, 90, 223114.

[17] a) H. Yoon, J. H. Ahn, P. W. Barone, K. Yum, R. Sharma, A. A. Boghossian, J. H. Han, M. S. Strano, Angew. Chem. 2011, 50, 1828; b) J. Zhang, M. P. Landry, P. W. Barone, J.-H. Kim, S. Lin, Z. W. Ulissi, D. Lin, B. Mu, A. A. Boghossian, A. J. Hilmer, A. Rwei, A. C. Hinckley, S. Kruss, M. A. Shandell, N. Nair, S. Blake, F. Sen, S. Sen, R. G. Croy, D. Li, K. Yum, J.-H. Ahn, H. Jin, D. A. Heller, J. M. Essigmann, D. Blankschtein, M. S. Strano, Nat. Nano 2013, 8, 959; c) E. S. Jeng, J. D. Nelson, K. L. J. Prather, M. S. Strano, Small 2010, 6, 40.

[18] S. Viswanathan, T. N. Narayanan, K. Aran, K. D. Fink, J. Paredes, P. M. Ajayan, S Filipek, P. Miszta, H. C. Tekin, F. Inci, U. Demirci, P. Li, K. I. Bolotin, D. Liepmann, V. Renugopalakrishanan, Mater. Today 2015, 18, 513.

[19] J. Hayes, P. P. Peruzzi, S. Lawler, Trends Mol. Med. 2014, 20, 460.

[20] I. Anosova, E. A. Kowal, M. R. Dunn, J. C. Chaput, W. D. Van Horn, M. Egli, Nucleic Acids Res. 2016, 44, 1007.

[21] J. D. Sander, J. K. Joung, Nat. Biotech. 2014 32, 347

[22] B. Leader, Q. J. Baca, D. E. Golan, Nat. Rev. Drug Discov. 2008, 7, 21.

[23] J. Bos, G. Roelfes, Curr. Opin. Chem. Biol. 2014, 19, 135.

[24] J. D. Bloom, M. M. Meyer, P. Meinhold, C. R. Otey, D. MacMillan, F. H. Arnold, Curr. Opin Struct. Biol. 2005, 15, 447.

[25] X.-G. Zhu, S. P. Long, D. R. Ort, Ann. Rev Plant Biol. 2010, 61, 235.

[26] P. Bombelli, R. W. Bradley, A. M. Scott, A J. Philips, A. J. McCormick, S. M. Cruz, A. Anderson, K. Yunus, D. S. Bendall, P. J. Cameron, J. M. Davies, A. G. Smith, C. J. Howe, A. C. Fisher, Ener. Environ. Sci. 2011, 4 4690.

[27] J. P. Giraldo, M. P. Landry, S. M. Faltermeier, T. P. McNicholas, N. M. Iverson, A. A. Boghossian, N. F. Reuel, A. J. Hilmer, F. Sen, J. A. Brew, M. S. Strano, Nat. Mater. 2014, 13, 400.

[28] M. Gong, T. A. Shastry, Y. Xie, M. Bernardi, D. Jasion, K. A. Luck, T. J. Marks, J. C. Grossman, S. Ren, M. C. Hersam, Nano Lett. 2014, 14, 5308.

[29] a) J. O. Calkins, Y. Umasankar, H. O'Neill, R. P. Ramasamy, Ener. Environ. Sci. 2013, 6, 1891; b) M.-H. Ham, J. H. Choi, A. A. Boghossian, E. S. Jeng, R. A. Graff, D. A. Heller, A. C. Chang, A. Mattis, T. H. Bayburt, Y. V. Grinkova, A. S. Zeiger, K. J. Van Vliet, E. K. Hobbie, S. G. Sligar, C. A. Wraight, M. S. Strano, Nat. Chem. 2010, 2, 929 . 